\title{
Control Strategy for Automatic Transmission under the Condition of Rough Road Feature Recognition
}

\author{
Yulong Lei, Qingkai Wei, Xingzhong Li, Xuesong Zheng and Yao Fu \\ State Key Laboratory of Automotive Simulation and Control, Jilin University, 130022, Changchun, China
}

\begin{abstract}
To solve the cycle shifting problem caused by double-parameter shifting schedule on the rough road, it is need to recognize rough road and then take corresponding control strategy. Firstly, vehicle longitunal dynamic on rough road is analyzed, which verified the possibility using longitudinal acceleration to recognize rough road. Then longitudinal acceleration expressions on the rough road are analyzed, which proves the turbine speed change rate is effective on rough road recognition, and time domain analysis of turbine speed change rate is done. With the above analysis, a method combined with frequency of turbine speed change rate passing through the limits and receding horizon is used to recognize rough road. Finally, the fixed method of shift schedule on rough road is proposed based on the above passing through frequency, and control strategy of automatic transmission under the condition of road recognition is also given. Road experiments show that road roughness is effectively recognized and cycle shifting problem can be effectively solved.
\end{abstract}

\section{Introduction}

When vehicle runs on rough road, rough road excitation will cause signal interference of vehicle speed sensor. Thus traditional dual-parameter shift schedule is based on the driving state on the fine level road, which may cause the cycle shifting on the rough road. So it is significant to recognize the rough road and then change the control strategy.

The real time estimations of the rough road feature and shift schedule design are two key factors. Various approaches to identify the rough road are developed which are based on wheel acceleration sensors of Antilock Braking System (ABS) [1-3] or vehicle body vertical acceleration [4-5]. Toshiaki Tsuyam [1] analyzed the impact of the rough road on the angular acceleration and wheel acceleration and proposed the recognition method of rough road based on wheel acceleration. Kong propose "energy specific weight method" and "account the times crossing method" to identify road based on the timefrequency analysis of wheel speed acceleration, and then designed a real-time algorithm for road roughness feature recognition. Cui [6] analyzed road surface power spectral density and proposed a method based on BP neural network to detect road roughness. But these methods cannot solve the problem of rough road recognition for automatic transmission.

Various researches have done on fixing the shift schedule to avoid cycle shifting problem. Wang proposed the accelerator passivated control strategy; the accelerator signal was treated with step by step method. Liu Hongbo [7] adopted the strategy of limiting the highest gear. Li
Runze [8] fixed the shift schedule of tracked vehicle based on engine speed change rate.

This paper proposes a novel rough road feature recognition method for automatic transmission. Based on the feature, control strategy on the rough road is proposed and applied to an off road vehicle.

\section{Vehicle longitudinal dynamics on rough road}

To analyse the rough road's effect on powertrain system, we need to analyse the rough road's effect on speed signal from the view of vehicle longitudinal dynamics. Rough road's feature can be described as road surface with changing slope angle and rolling resistance coefficient. As Figure 1 shown, the rate of slope angle change should be in a small range, changing direction, randomly and unavoidable. Rolling resistance coefficient is determined by road roughness, coefficient of road adhesion and other factors.

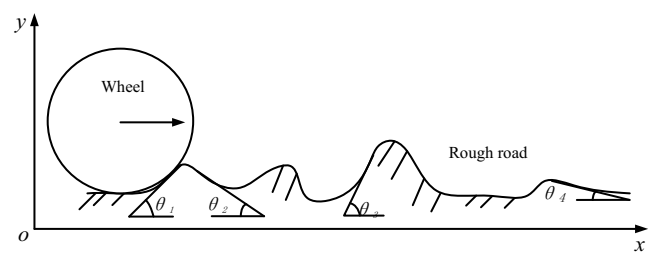

Figure 1. Schematic diagram of vehicle on rough road.

From the automotive running equations, acceleration can be right as the follow form: 
$\frac{d u}{d t}=\frac{1}{\delta m}\left(\frac{T_{t} i_{g} i_{0} \eta_{T}}{r}-m g \tilde{f} \sin \tilde{\theta}-m g \cos \tilde{\theta}-\frac{C_{D} A u_{a}^{2}}{21.15}\right)$

In the formula:

$\tilde{f}$ - changing rolling resistance coefficient on rough road;

$\tilde{\theta}$ - changing slope angle on rough road;

$u$ - vehicle speed;

$m$ - vehicle mass;

$\delta$ - rotating mass conversion factor;

$T_{t}$ - turbine torque;

$i_{g}, i_{0}$ - gear ratio of transmission and main reducer;

$\eta_{T}$ - transmission efficiency;

$A$ - air resistance coefficient;

$C_{D}$ - automotive frontal area;

$r$ - tyre rolling radius.

On the rough road, two factors mainly impacts on change of driving resistance: one is the change of slop angle which leads to the change of rolling resistance and climbing resistance; the other is the change of wheel's contact area with road surface and road adhesion coefficient which lead to the change of rolling resistance. If the shift process is not started, turbine torque will not have obviously fluctuation, so the changes of driving resistance will finally lead to the fluctuant acceleration.

\section{Rough road feature recognition method}

\subsection{Decision of recognition signal}

According to the above analysis, longitudinal acceleration can be used to identify rough road. For automatic transmission, longitudinal acceleration's manifestation includes turbine speed change rate and vehicle speed change rate.
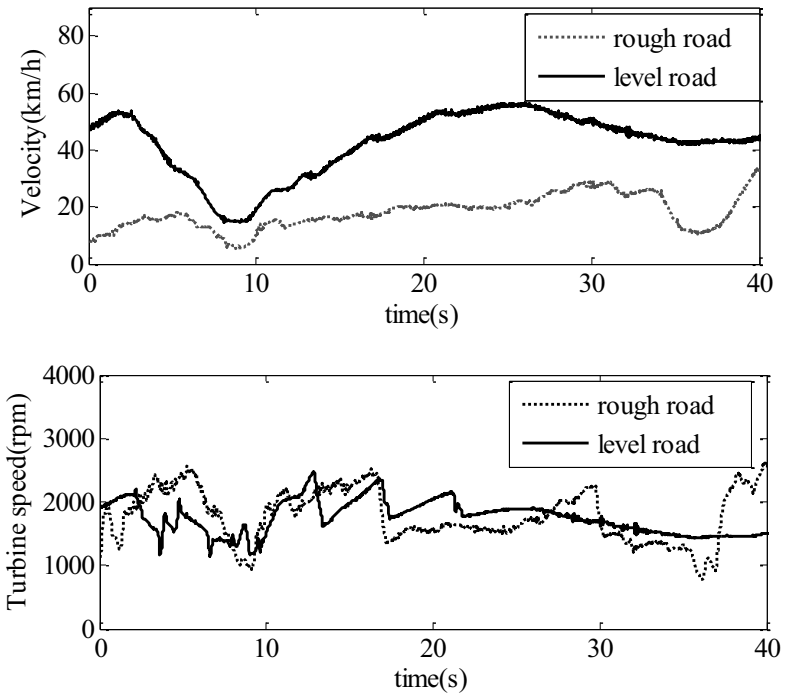

Figure 2. Comparison of vehicle speed and turbine speed on rough road and level road.

For vehicle speed change rate, vehicle speed should be got firstly. Vehicle speed can be calculated by output speed sensor. Vehicle speed on rough road is usually lower than that on level road, as vehicle speed change rate is the speed difference of current time and previous time. If vehicle speed is high, speed difference will be large; otherwise speed difference is small. So vehicle speed is not effective on recognizing acceleration caused by road surface, that is to say different speed disturbs the recognition of vehicle speed change rate.

For turbine speed change rate, value range of turbine speed is smaller, namely different speed will not disturb the recognition of turbine speed change rate. Besides, small obstacles, such as small stone, will not disturb turbine speed obviously. Figure 2 shows the comparison of vehicle speed and turbine speed on rough road and level road.

\subsection{Time-domain signal analysis}

Based on the above analysis, turbine speed is more suitable for recognizing rough road than vehicle speed. Figure 3 is the comparison of turbine speed change rate on rough road and level road.
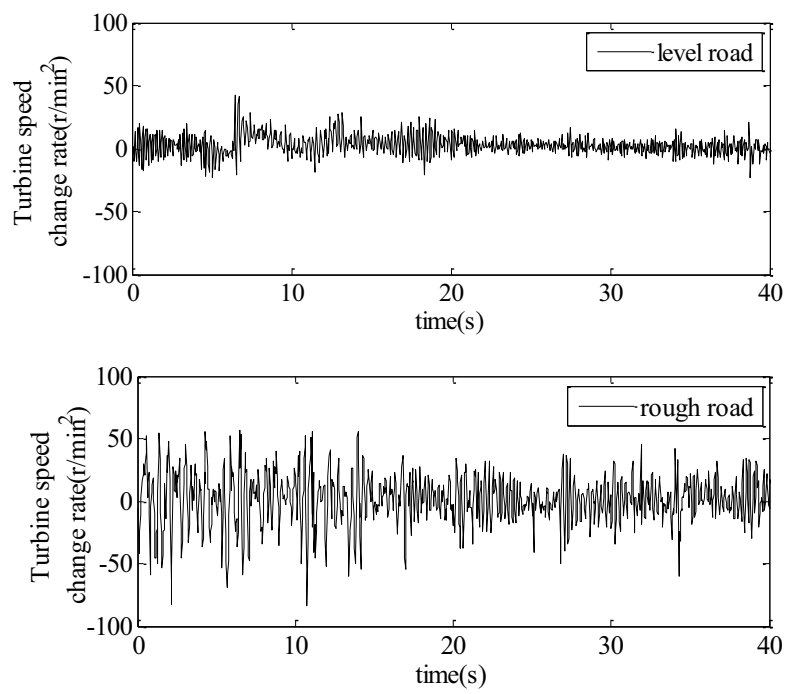

Figure 3. Comparison of turbine speed change rate on rough road and level road.

It can be seen from Figure 3 that turbine speed change rate is larger than change rate on level road. Table 1 is the statistical analysis of these two change rates.

Table 1. Statistical analysis of turbine speed change rate on rough road and level road.

\begin{tabular}{|c|c|c|}
\hline Road surface & Level road & Rough road \\
\hline Maximum Value & 41.2 & 59.3 \\
\hline Minimum Value & -21.9 & -85.6 \\
\hline Average absolute value & 16.8 & 37.6 \\
\hline Standard deviation & 7.8965 & 21.3527 \\
\hline
\end{tabular}

As Table 1 shown, fluctuation range of turbine speed change rate on rough road is greater than that on level road. So we can assign a limit value of turbine speed change rate. Then the times passing through the limit 
value is recorded in a count cycle and the times is defined as passing through frequency. The passing through frequency is used as numerical feature of turbine speed change rate in time domain. The statistical law of passing through frequency after 10-cycle experiments is as Figure 4 and Table 2 shown. So a threshold value is assigned, when passing through frequency is larger than the threshold value, this road is determined to be rough road; otherwise, this road is level road. Furthermore, a larger passing through frequency means that the vehicle is on a rougher road.

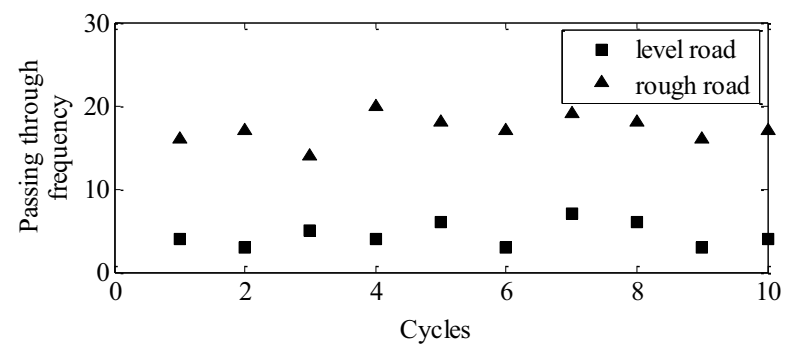

Figure 4. Passing through frequency of 10-cycle.

Table 2. Statistical analysis of passing through frequency.

\begin{tabular}{|c|c|c|}
\hline Road surface & Level road & Rough road \\
\hline Maximum Value & 3 & 14 \\
\hline Minimum Value & 7 & 20 \\
\hline Average absolute value & 4.5 & 17.38 \\
\hline Standard deviation & 1.4337 & 1.6865 \\
\hline
\end{tabular}

\subsection{Feature recognition method based on receding horizon}

From the above analysis, statistical law of passing through frequency agrees with the actual road condition, it can be seen that this method is effective to recognize rough road. But if the count cycle is set short, a larger statistical error may occur; while a long period affects its real-time quality. So a receding horizon is designed, which is based passing through times receding time window instead of the current period. Figure 5 is the flowchart of real-time road surface recognition algorithm.

Real-time algorithm introduction is as follows:

(1) Set the initial value of turbine speed change rate limit value $\pm \alpha$, threshold of passing through frequency $\beta$, flag of rough road RFlag, receding window size $K$, count cycle $T$, road roughness sum counter $R, R_{\text {count }}[i]=0$, $i=1,2, \ldots, \mathrm{K}$.

(2) Caculate $R_{\text {count }}[i] . R_{\text {count }}[i]=R_{\text {count }}[i+1], i=1,2, \ldots$, $\mathrm{K}-1, \quad R=\sum R_{\text {count }}[i], i=1,2, \ldots, \mathrm{K}-1$.

(3) When turbine speed change rate $\Delta n_{t}$ exceeds $\pm \alpha$ in a count cycle, counter $R_{\text {count }}[K]=R_{\text {count }}[K]+1$.

(4) When a count cycle finish, $R=R+R_{\text {count }}[K]$.

(5) If roughness counter $R$ is greater than $\beta$, road surface in this count cycle is considered to be rough road, set $R$ Flag $=1$; otherwise is level road, set $R$ Flag $=0$.

(6) Reset counter $R, R_{\text {count }}$, enter the next count cycle. Go to step (2).

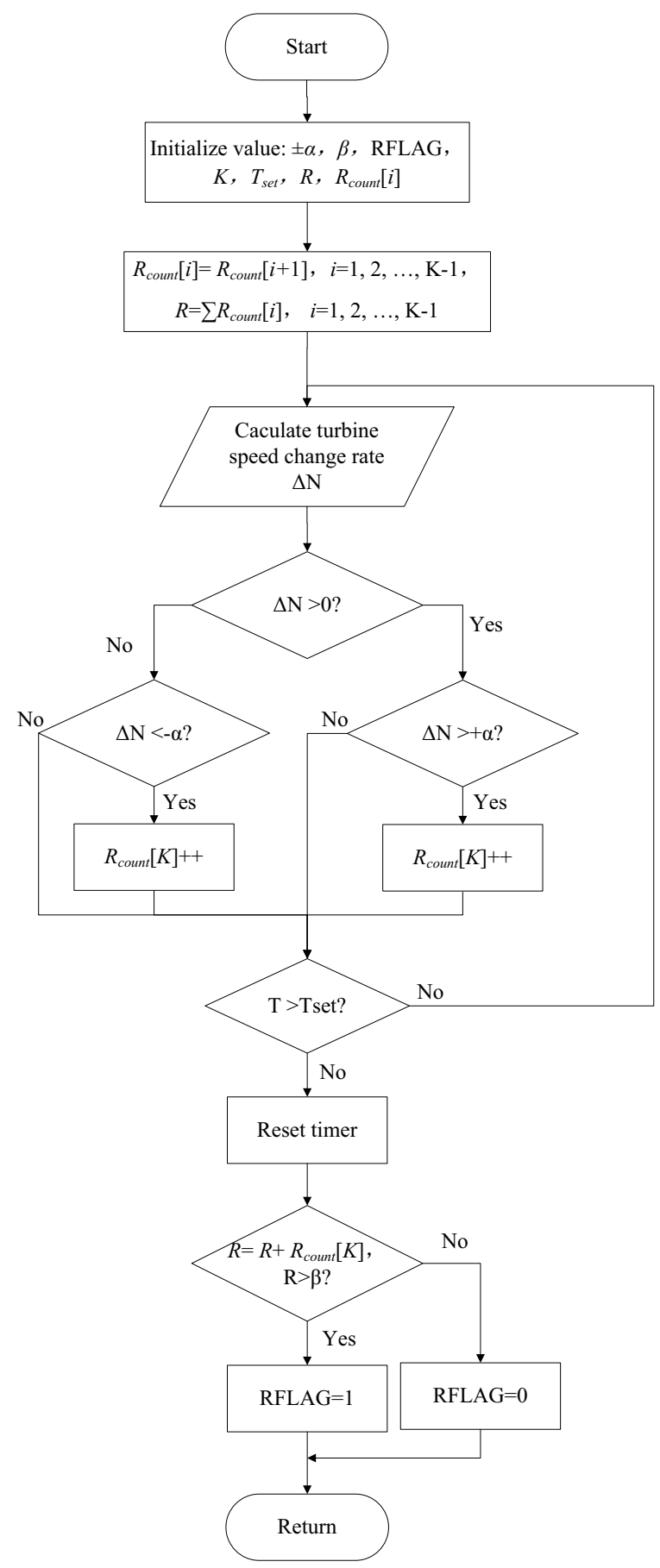

Figure 5. Flowchart of algorithm based on receding horizon.

\section{Control strategies under the condition of road recognition}

\subsection{Shift schedule on rough road}

Traditional shift schedule is based on vehicle speed and accelerator pedal position as control parameters and established on level road. But on the rough road, driver's pedal is usually in a fluctuant position, thus this fluctuation is not driver's intension, as figure 6 shows, so accelerator pedal position is not suitable for shifting schedule's control parameter. 


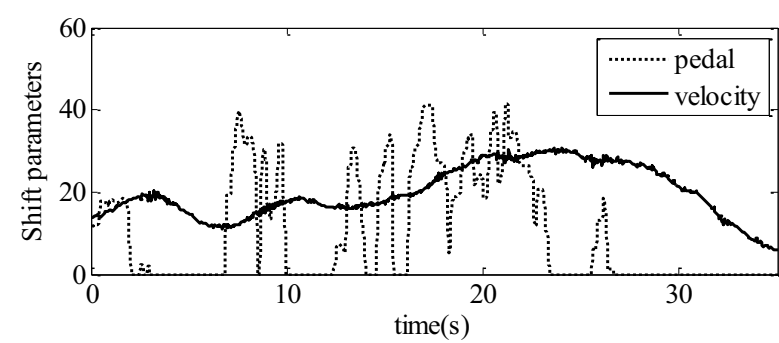

Figure 6. Vehicle speed and acceleration pedal on rough road.

Based on the above analysis, this article use turbine speed change rate passing through frequency and vehicle speed as shift schedule's control parameter, meanwhile limits the highest gear according to this passing through frequency. For an off-road vehicle with 6-speed automatic transmission in this research, the fixed shift schedule is as Figure 7 shown.

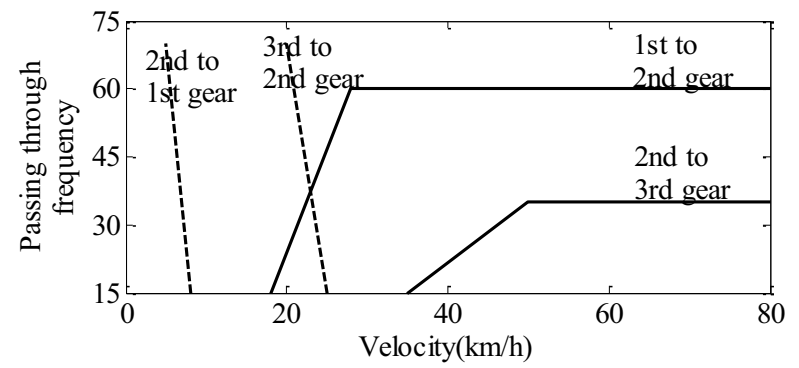

Figure 7. Shift schedule based on vehicle speed and passing through frequency.

If the passing through frequency is less than $30 \mathrm{~Hz}$, the current road surface is seen as a common rough road, maximum allowable gear is 3rd gear; otherwise if the passing through frequency is less than $60 \mathrm{~Hz}$, the current road surface is seen as a highly rough road; if the passing frequency is greater than $60 \mathrm{~Hz}$, upshift is forbidden. At the same time, if the passing through frequency is higher, upshift point of vehicle speed is higher but downshift point is lower, the reason is to avoid cycle shifting lead by vehicle speed fluctuation.

\begin{tabular}{l}
\hline Rough road: \\
1 Disengage lock-up clutch, torque converter \\
works in hydraulic condition \\
2 Shift schedule based on velocity and passing \\
through frequency
\end{tabular}

Figure 8. Control strategy under the condition of road feature recognition.

\subsection{Architecture of control strategy under the road condition recognition}

When rough road is recognized, transmission control unit (TCU) needs to adopt corresponding control strategy to ensure the vehicle riding comfort, meanwhile recognize whether the vehicle leaves rough road so as to quit the control strategy on rough road. The flow chart is as Figure 8 shown.

When vehicle travels on rough road, besides changing the shift schedule, lock-up clutch should be disengaged to make the torque converter working in hydraulic state, so as to improve the vehicle ride performance. When TCU recognize that passing through frequency is less than threshold value and difference speed of engine speed and turbine speed is less than set value, the vehicle is considered to leave rough road.

\section{Analysis of the vehicle tests results}

\subsection{Rough road recognition method tests}

Apply the recognition method on actual road surface recognition, vehicle tests results is as Figure 9 shown.
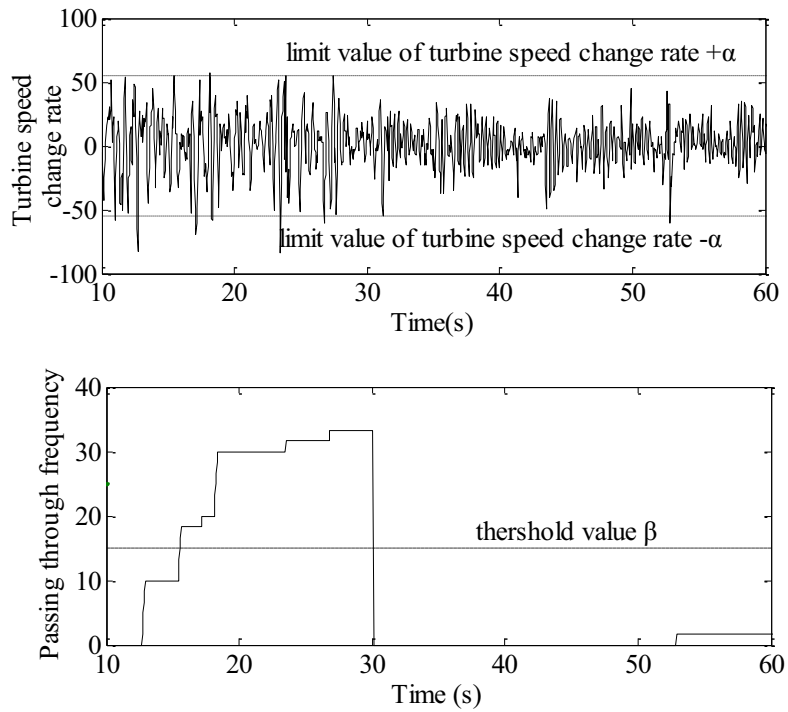

Figure 9. Validation of rough road recognition.

It can be seen from figure 9 that passing through frequency exceeds threshold value from 15 s that is to say vehicle begins to run on rough road. The vehicle quits from 30 s as the passing through frequency is less than threshold value. The recognition result meets the real road surface.

\subsection{Control strategy on rough road tests}

Figure 10 is the gear situation comparison of tradition shift schedule and proposed shift schedule.

In the traditional shift schedule, vehicle and pedal acceleration reaches upshift point at about $2 \mathrm{~s}$, transmission shifts to 3rd gear. But as the impact of rough road transmission suddenly starts down-shift process, 
which is lead to cycle shift problem. But the proposed method avoids this problem, as the upshift point of vehicle speed is higher. Vehicle tests prove the effectiveness of the propose shift schedule.
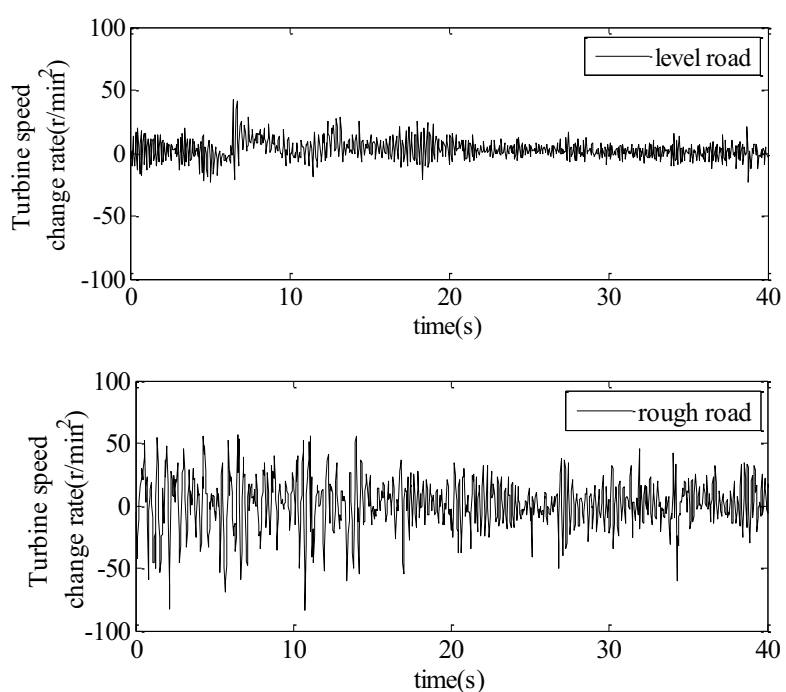

Figure 10. Comparison of turbine speed change rate on rough road and level road.

\section{Conclusions}

(1) With the dynamic analysis of vehicle on rough road, longitudinal acceleration is effective on rough surface recognition. Furtherly, turbine speed change rate is a kind of more effective longitudinal acceleration signal.

(2) By time-domain analysis of turbine speed change rate, passing through frequency method is proposed.
Combined with receding horizon, this method is effective on rough road recognition.

(3) Shift schedule fixed method is researched. Both considering vehicle's passing ability and avoiding cycle shifting, shift schedule based on passing through frequency and vehicle speed is proposed. This method is well performed on the road test.

\section{Acknowledgements}

This study was supported by the National Natural Science Foundation of China (Grant No. 51575220).

\section{References}

1. T. Tsuyama, K. Nobumoto, K. Sotoyama, T. Onaka, F. Kageyama, \& H. Okazaki. EP US5117934A (1992).

2. H. Zhang, B. Chen, T. Zhang, D. Liu, Automotive Engineering, 24 (2002)

3. L. Kong, J. Song, Y. Yan, J. Shen, CHIN J MECH ENG, 43 (2007).

4. R. Wang, X. J, G. Wang, Automotive Engineering, 30 (2008)

5. D. Cui, C. Zhang, D. Han, Computer Simulation, 31 (2014)

6. H. Wang, H. Liu, W. Zhang, H. Chen, J B INST TECHNO, 29 (2009)

7. H.B. Liu. Changchun: Jilin University(2012).

8. R.Z. Li. Beijing: Beijing Institute of Technology (2015) 